\title{
ON THE PHENOMENOLOGICAL THEORY OF MAGNETIC STORMS
}

\section{A.V. Guglielmi}

Schmidt Institute of Physics of the Earth, RAS, Moscow, Russia, guglielmi@mail.ru

This article addresses methodical issues concerning the modeling of the $D_{\text {st }}$ variation in a geomagnetic storm. We describe the so-called RBM (Russell - Burton - McPherron) model representing an ordinary differential equation with solutions simulating the relation between the $D_{\text {st }}$ variation and the azimuthal component of the interplanetary electric field. Special attention is paid to the threshold nature of $D_{\text {st }}$ variation excitation. We would like to emphasize the necessity of stochastic extension of the RBM model by taking into account fluctuations inherent to any physical system. The integral representation of a $D_{\text {st }}$ variation bifurcation diagram is given. It enables us to account for the effect of fluctuations that eliminate the diagram root singularity and cause a threshold point shift. The $D_{s t}$ variation is shown to be typical of the wide class of threshold phenomena similar to second-order phase transitions. We draw an analogy with threshold phenomena in Earth's magnetosphere, atmosphere, and lithosphere. In addition, we briefly discuss the issue about soft and hard passages through the threshold, as well as about explosive instability in geophysical media.

Keywords: Magnetosphere, phase transition, bifurcation, fluctuations, explosive instability, atmosphere, lithosphere.

\section{INTRODUCTION}

The solar wind and interplanetary magnetic field surprisingly constantly cause usually smooth and sometimes abrupt changes in Earth's magnetosphere. This paper considers one example of such changes in the most simplified form. The case in hand is a magnetic storm. Attention here focuses on a storm-time variation $\left(D_{\text {st }}\right)$ representing the most important magnetic storm effect [Nishida, 1980].

The phenomenological theory of $D_{\text {st }}$ variation is proposed in [Burton et al., 1975]. It has the form of the first-order differential equation with solutions simulating the relationship between the $D_{\text {st }}$ variation and the azimuthal component of the interplanetary electric field. We call this equation RBM (Russell Burton - McPherron) model as in [Guglielmi, Pokhotelov, 1996]. In [Guglielmi, 1988], this model was refined, and in [Guglielmi et al., 1989] the proposed modification was experimentally justified.

The RBM model falls into the type of so-called toy models that are widely used in theoretical physics and astrophysics for describing very intricate and/or not entirely understood processes. The magnetic storm complexity needs little comment. The complexity itself justifies the use of the phenomenological reduction and extremely simple mathematical tools to formalize observations without 
making unmotivated hypotheses ad hoc. However, in addition to the complexity there is an incompletely solved problem, namely, the problem of reconnection of interplanetary and geomagnetic field lines. The reconnection produces ring current sources generating the $D_{\mathrm{st}}$ variation [Nishida, 1980]. Reconnection physics is not fully understood [Goldstein, 2001], but the phenomenology of reconnection is relatively simple [Dungey, 1961]. This very circumstance in due course opened up the possibility for building the RBM model.

The present paper is methodological and debatable. Section 1 describes the modified RBM model. Special attention here is paid to the threshold character of $D_{\text {st }}$ variation excitation. Section 2 highlights the need for stochastic extension of the RBM model by allowing for fluctuations influencing the formation of $D_{\mathrm{st}}$ variation. Section 3 gives an integral representation of bifurcation diagram of a ring current source. This facilitates the consideration of the effect of fluctuations that eliminate the root singularity at a threshold point. Section 4 shows that the $D_{\text {st }}$ variation belongs to a broad class of threshold phenomena akin to second-order phase transitions. We draw an analogy with threshold phenomena in Earth's magnetosphere, atmosphere and lithosphere. In addition, we briefly discuss the issues about soft and hard passages of physical systems through the threshold, as well as about explosive instability in geophysical media. The final section succinctly summarizes analysis findings.

\section{RBM MODEL}

The modified RBM model looks as follows. Let $E(t)$ be an azimuthal component of the interplanetary electric field, and $q(t)$ be a ring current source responsible for a $D_{\mathrm{st}}$-variation, with $q \geq 0$. Instead of $D_{\text {st }}$, it is convenient to employ

$$
D=a+b p^{1 / 2}-D_{\mathrm{st}},
$$

proportional to the ring current intensity. Here $p$ is the solar wind dynamic pressure. In what follows, the numerical values of $a$ and $b$ are not needed. The evolution of $D(t)$ is described by the equation

$$
d D / d t=q-D / \tau,
$$

where $\tau$ is the time of ring current decay. Accurate within the proportionality coefficient, the dependence of $q$ on $E$ is as follows: $q=0$ if $E<E_{\mathrm{c}}$, and $q=\left(E-E_{\mathrm{c}}\right)^{1 / 2}$ if $E \geq E_{\mathrm{c}}$. Here $E_{\mathrm{c}}$ is the critical (threshold) value of the azimuthal component of the interplanetary electric field (see Figure 1). The threshold character of the $q$ dependence on $E$ suggests that we deal with instability. This is also confirmed by physical considerations on the geomagnetic and interplanetary magnetic field line reconnection. This reconnection appears to induce the $D_{\mathrm{st}}$ variation.

Introduce the potential $U(q, E)$ and consider the dynamic system

$$
d q / d t=\partial U / \partial q,
$$

that provides metaphorical understanding of the origin of the $q$ dependence on $E$. Redefine the dimensions $t$ and $q$ in such a way as to avoid overloading the description with unnecessary proportionality coefficients, and assume

$$
U=\left(E_{\mathrm{c}}-E\right) q^{2} / 2+q^{4} / 4 .
$$




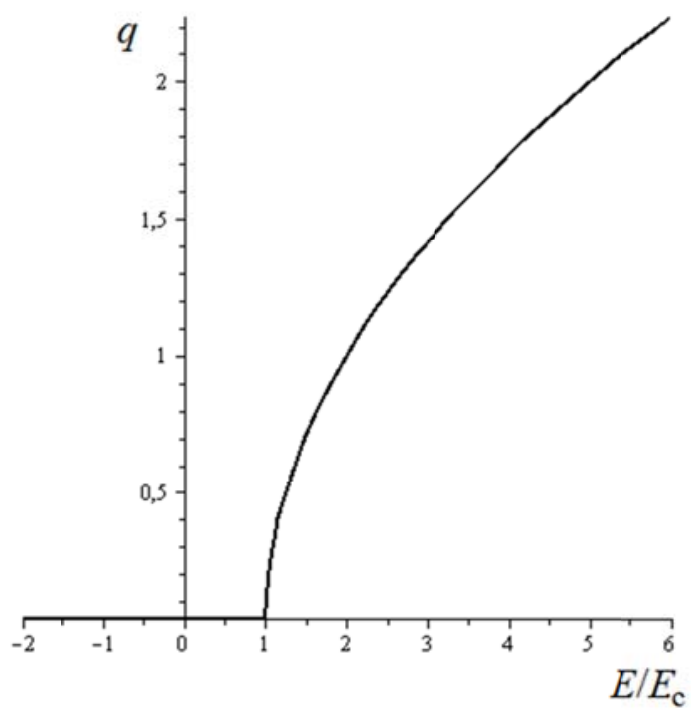

Figure 1. The source of the $D_{\text {st }}$ variation in the geomagnetic field as a function of the azimuthal component of the interplanetary electric field within the modified RBM model

Remind that in the theory of phase transitions and catastrophe theory, $q$ is called the order parameter; $E$, the control parameter; and $E=E_{\mathrm{c}}$, the bifurcation point (see, e.g., [Gilmore, 1984]). The $q(E)$ dependence is sometimes termed as the bifurcation diagram.

It is easy to ensure that in equilibrium (3) and (4) yield the above $q(E)$ dependence. Indeed, if $E<E_{\mathrm{c}}$ there exists one equilibrium point $q=0$, and it is stable. Increasing $E$ leads to bifurcation, and with $E>E_{\mathrm{c}}$ there appear two equilibrium points with unstable $q=0$ and stable $q=\left(E-E_{\mathrm{c}}\right)^{1 / 2}$. It is apparent that the order parameter is equal to the square root of supercriticality. By comparison, point out that in the classical RBM model the $q(E)$ dependence is linear at $E>E_{\mathrm{c}}$ [Burton et al., 1975]. Dynamic description (3) gives the linear dependence if the second term in the right side of (4) is replaced by $q^{3} / 3$. Then, dynamic system (3) has quadratic nonlinearity instead of cubic one. This is theoretically possible, but the choice of potential (4) fits the observations better [Guglielmi et al., 1989].

\section{FLUCTUATIONS}

So, the $q(E)$ dependence is threshold. The theory of dynamic systems suggests that, going toward the threshold, fluctuations, inherent to any real system, begin to play a more important role [Gilmore, 1984; Horsthemke, Lefever, 1984]. There are two reasons why we should stochastically extend the RBM model and examine the influence of fluctuations on the bifurcation diagram in the vicinity of a threshold point. First, the fluctuations smooth over the threshold, thus eliminating the physically unjustified singularity of $q(E)$ at $E=E_{\mathrm{c}}$. Second, the fluctuations actually displace the threshold. This circumstance should be taken into account to interpret the experimentally measured values of $E_{\mathrm{c}}$.

The search for stochastic extension of the RBM model raises a question as to which of the two basic equations - (2) or (3) - is of main interest for the above formulated problem. To be sure, the inclusion of 
fluctuations in nonlinear equation (3) is more interesting than in linear equation (2). Treating Equation (3), put ourselves the second question: what fluctuations - additive or multiplicative - should first be selected for the analysis? If it is a model with additive fluctuations, then to the right side of (3) we should add a Langevin source. The origin of such a fluctuation source is still not quite clear. The introduction of the Langevin source would be a typical hypothesis ad hoc. At the same time, the idea of the existence of a multiplicative source needs no additional hypotheses. It naturally stems from the structure of $U(q, E)$. The control parameter $E$ undergoes fluctuations associated with the presence of MHD waves in the solar wind. Since the control parameter is involved in (4) as $E q^{2}$, the MHD waves generate the multiplicative fluctuations that should be accounted for in the stochastic extension of (3).

The third question refers to the origin and spectral and temporal structure of the control-parameter fluctuations. Here we give only general information. Before a near-Earth shock front there permanently exist turbulent pulsations in the electromagnetic field with their spectrum covering a frequency range from $\mathrm{mHz}$ to several $\mathrm{kHz}$ [Russell, Hoppe, 1983]. These pulsations arise from the nontrivial interaction of solar wind ions and electrons with the shock front. Wave disturbances also occur in the free solar wind. They propagate from the Sun as Alfvén waves and also appear in interplanetary space between the Sun and Earth due to fire-hose and other instabilities of interplanetary plasma.

\section{INTEGRAL REPRESENTATION}

In this case, the problem is to find an appropriate form for the stochastic extension of the RBM model. Consider Figure 1 once again. This figure indicates that in dynamic representation the $q$ value near the threshold is proportional to the square root of the interplanetary electric field $E$. Notice an interesting analogy: in some cases the nuclear reaction cross-section is also proportional to the square root of energy of a particle bumping into a nucleus. This observation suggested the possibility of utilizing the procedure proposed in [Migdal, 1975].

Assume first that there are no fluctuations in the interplanetary electric field. Introduce the integral representation for $q(E)$ :

$$
q(E)=\int_{E_{\mathrm{c}}}^{\infty} \delta\left(E^{\prime}-E\right) \sqrt{E^{\prime}-E_{\mathrm{c}}} d E^{\prime} .
$$

It is readily seen that it is no more than a new analytical expression for the function shown in Figure 1. In actual fact, from (5) it follows that $q=0$ if $E<E_{\mathrm{c}}$ and $q=\sqrt{E-E_{\mathrm{c}}}$ if $E>E_{\mathrm{c}}$ as is shown in Figure 1.

However, under real conditions, the value of the interplanetary electric field is far from being fixed. It fluctuates about a mean value of $E$. Integral representation (5) suggests that we should change the delta function to a relevant distribution function:

$$
q(E)=\int_{E_{\mathrm{c}}}^{\infty} f\left(E^{\prime}-E\right) \sqrt{E^{\prime}-E_{\mathrm{c}}} d E^{\prime} .
$$


Given the Gaussian distribution

$$
f(x)=\frac{1}{\sigma \sqrt{2 \pi}} \exp \left[-\frac{1}{2}\left(\frac{x}{\sigma}\right)^{2}\right]
$$

with the standard deviation $\sigma$, (6) yields

$$
q(E)=\sqrt{\frac{\sigma}{2}} \exp \left[-\left(\frac{E-E_{\mathrm{c}}}{2 \sigma}\right)^{2}\right] D_{-3 / 2}\left(\frac{E_{\mathrm{c}}-E}{\sigma}\right),
$$

where $D_{-3 / 2}$ is a parabolic cylinder function.

Analyze Formula (8). Using the asymptotic form of the parabolic cylinder function [Gradshteyn, Ryzhik, 1962], set $\sigma=$ const and find that $q=0$ with $E \rightarrow-\infty$ and $q=E^{1 / 2}$ with $E \rightarrow+\infty$ asi was expected from the derivation of Formula (8). If, however, $E=$ const and $\sigma \rightarrow 0$, then (8) yields the bifurcation diagram drawn in Figure 1. Thus, in the three extreme cases there is a one-to-one correspondence between stochastic and deterministic RBM models. At small but finite $\sigma$, the influence of fluctuations is most pronounced in a small vicinity of the threshold. Figure 2 illustrates this position at $\sigma=0.2$. It is clear that the root singularity disappeared, i.e. the threshold was lowered. Besides, it slightly shifted to the left.

\section{DISCUSSION}

The $D_{\text {st }}$ variation falls into a certain class of geophysical phenomena whose characteristic feature is that the function of system status is continuous, but the control parameter derivative of the function experiences a jump when passing over the threshold of the control parameter.

In statistical physics, this corresponds to second-order phase transitions [Landau, Lifshits, 2005]. The threshold singularity arose from idealization, and the idealization was that in our theory, presented by Equations (2)-(4), we made a phenomenological reduction in an indefinitely large number of degrees of

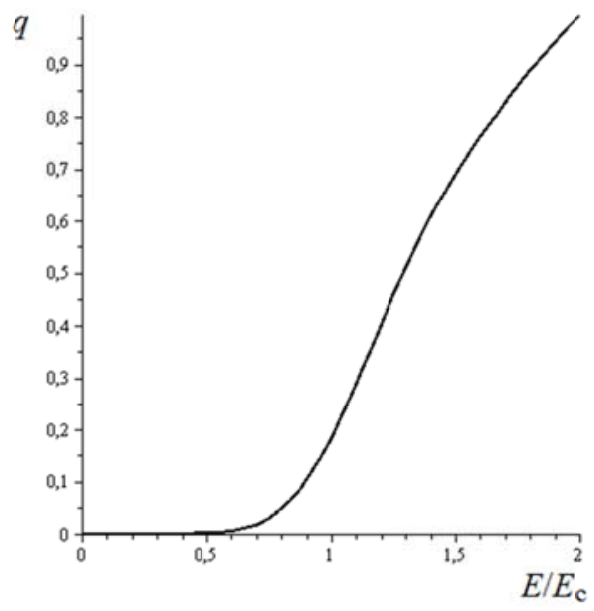

Figure 2. Smoothing and shift of the threshold under the action of multiplicative fluctuations of the control parameter 
freedom of the magnetosphere and solar wind. If necessary, neglected degrees of freedom can be included in the theory as additive or multiplicative noises. This situation is by no means unique. Geophysics deals with many threshold events akin to second-order phase transitions. Several typical examples are given below. In each of them, we can allow for fluctuations through the integral representation of bifurcation diagram just as we have made in the stochastic extension of the RBM model of $D_{\mathrm{st}}$ variation.

As a well-known example we can point to self-excitation of ion-cyclotron waves in Earth's radiation belt. In this case, it is possible to explicitly find the threshold and determine the evolution of the system in crossing the threshold from fundamental equations of plasma electrodynamics [Guglielmi, Pokhotelov, 1996; Kangas et al., 1998]. However, many other cases defy sequential analysis based on general principles. Instead, we have to build phenomenological models from observations and physical considerations. A typical example is the model of wind-driven generation of atmospheric electricity at the drifting ice station North Pole - 22 [Guglielmi et al., 1979]. The control parameter is a wind speed. The critical speed of $4 \mathrm{~m} / \mathrm{s}$ was found from observations and was substantiated by general considerations for electrification of snow and ice particles in airflow. Another example refers to the liquid motion in pores and cracks of rocks. The velocity $V$ of the fluid flow in porous media is usually described by Darcy's law $V=K_{\mathrm{D}} I$ where $K_{\mathrm{D}}$ is a linear filtration coefficient and $I$ is a pressure gradient [Collins, 1964]. However, experience has shown that there are filtration mechanisms that require us to make corrections to Darcy's linear law. In particular, the effective permeability coefficient $K(I, \delta I)$ with $I=0$ is

$$
K(0, \delta I)=\left.\frac{d V}{d I}\right|_{I=0}=K_{\mathrm{D}}\left[1-\operatorname{erf}\left(\frac{1}{\sqrt{2}} \frac{I_{\mathrm{c}}}{\delta I}\right)\right]
$$

where $\delta I$ denotes pressure gradient fluctuations, $I_{\mathrm{c}}$ is the so-called Zlochevskaya's threshold [Guglielmi, 2002]. It means that the permeability coefficient calculated in a laboratory on a real rock sample will be below the Darcy coefficient $K_{\mathrm{D}}$.

Experience of phenomenological modeling of threshold phenomena suggests that sometimes the common form of a geophysical process indicates the form of a transition curve and even enables us to guess the form of a differential equation describing the process as in the modeling of the $D_{\mathrm{st}}$ variation. The right choice of the evolution equation is particularly important in trying to go beyond the scope of the class of phenomena, we considered, that are similar to second-order phase transitions. In some cases, the simple equation for a damped oscillator with quadratic nonlinearity

$$
d x / d t+\alpha x=\beta x^{2}
$$

may be a useful initial model in answering the question about the type of the dynamic system whose evolution is observed in the experiment. Illustrate the above with an example of self-excitation of ioncyclotron oscillations in Earth's radiation belt. In this case, $x$ is the intensity of oscillations, $\alpha$ is the control parameter dependent on proton anisotropy in the radiation belt with a threshold value $\alpha=0$ [Guglielmi, Pokhotelov, 1996]. The dynamic system type is defined by the sign of $\beta$. It is normally assumed that $\beta<0$ [Kangas et al., 1998]. If so, we have a self-oscillation system with soft self-excitation. The system status $x$ continuously depends on $\alpha$, but the $\alpha$ derivative of $x$ experiences a jump at a threshold 
point. In other words, if $\beta<0$, then the self-excitation of ion-cyclotron oscillations in the radiation belt should be assigned to the same class as the $D_{\mathrm{st}}$ variation. Note, however, that the hypothesis on hard selfexcitation that occurs at $\beta>0$ cannot be ruled out for now. There are observations indirectly indicating that the oscillation intensity $x$, and not only the $\alpha$ derivative of $x$, discontinues at the critical point $\alpha=0$. The question about the self-excitation mode in the radiation belt is underexplored and controversial.

The search for and study of systems with hard self-excitation in geophysical media are of obvious interest. Of particular interest is the case of explosive instability with which $x \rightarrow \infty$ over a finite time interval. Equation (10) simulates the explosive instability at $\alpha>0, \beta>0$, and sufficiently large initial values of $x_{0}$. Let, for simplicity, $x_{0}>>\alpha / \beta$. Then

$$
x(t)=x_{0}\left(1-t / t_{0}\right)^{-1},
$$

where $t_{0}=\left(\beta x_{0}\right)^{-1}$. The value $x \rightarrow \infty$ if $t \rightarrow t_{0}$. It means that in respect, for example, to a rigid body the explosive instability inevitably leads to its fracture in a time $t_{0}$. We can assume that the explosive instability occurs sporadically during formation of the main rupture in an earthquake focus, magnetic field line reconnection in the neutral layer of the geomagnetic tail, and most likely in many other natural phenomena.

Turn once more to Equation (10). Omit the second term from the left side, set $\beta=-1$ and write the equation as $d n / d t=n^{2}$. This equation is of no concern to physics of threshold phenomena. It describes, for example, the recombination that causes a monotonous decrease in the number $n$ of pairs of oppositely charged particles in ionospheric plasma. However, we call your attention to an interesting circumstance. The solution of $n \sim t^{-1}$ simulates Omori's empirical law that circumscribes a monotonous decrease in the number of aftershocks with the course of time after a strong earthquake [Omori, 1894]. Perhaps it is merely a coincidence. Yet it would be interesting to try to see sense in the analogy between the dynamics of aftershocks and the recombination of oppositely charged particles in ionospheric plasma. In this regard, we can make the following comments.

It is well known that the quadratic nonlinearity in the recombination equation appears due to binary collisions at equal concentrations of positively and negatively charged particles (the equality is very accurate in plasma). If we attach any significance to our attempt, we should perhaps pay attention to the remote resemblance between the pair of oppositely charged particles in plasma and the pair of sides of fault in the earth's crust. Continuing this line of reasoning, we have to make the next step and with all necessary reservations draw an analogy between the mean waiting time of collision of the pair of oppositely charged particles and the mean time of activation of the pair of adjacent sides of fault. Thus we can attempt to construct a recombination model of aftershock sequence.

To be sure, these considerations are incomplete. They have not advanced our understanding of earthquake physics so far. And it is not inconceivable that it was merely a coincidence. In this case, the equation $d n / d t=n^{2}$ has to be considered only as an equivalent form of Omori's empirical law. However, even in this case an additional opportunity arises to seek for corrections to Omori's law. For example, if in 
$d n / d t=-n^{2}$ the diffuse term is taken into account, i.e. is written as

$$
\partial n / \partial t=-n^{2}+D \nabla^{2} n,
$$

then the transition to the classical equation FKPP well-known in mathematics and biology is immediate [Fisher, 1937; Kolmogorov et al., 1937]. Using the variety of solutions of the FKPP equation, we extend our possibilities of searching for adequate models of spatio-temporal distribution of aftershocks.

\section{CONCLUSION}

This paper has addressed issues of the phenomenological modeling of the $D_{\text {st }}$ variation. We described the modified RBM model and posed a question concerning the need for stochastic extension of this model. Attention was given to the formal similarity between threshold features in the theory of nuclear reactions and in the theory of $D_{\text {st }}$ variation. This allowed us to use the integral representation of bifurcation diagram well-known in the quantum theory. Besides, this enabled us to account for the influence of fluctuations that eliminate the root singularity of the diagram and cause a threshold point shift. I think that it is especially important that the $D_{\text {st }}$ variation belongs to the broad class of magnetospheric, atmospheric, and lithospheric phenomena akin to second-order phase transitions.

I thank A.S. Potapov for taking an interest in this work and helpful comments. The study was carried out as part of RFBR projects 15-05-00491 and 16-05-00056.

\section{REFERENCES}

Burton R.K., McPherron R.L., Russell C.T. An empirical relationship between interplanetary conditions and $D_{\text {st }}$ J. Geophys. Res. 1975, vol. 80, no 31, pp. 4204-4214.

Collins R.E. Flow of fluids through porous materials. New York, Reinhold Publishing Co., 1961, 270 p. (in English). (Russian edition: Kollinz R. Techeniya zhidkostei cherez poristye materialy. Moscow, Mir Publ., 1964, 352 p.).

Dungey J.W. Cosmic Electrodynamics. Cambridge, Cambr. Univ. Press, 1958, 183 p. (In English). (Russian edition: Danzhi Dzh. Kosmicheskaya elektrodinamika [Cosmic Electrodynamics]. Moscow, Gosatomizdat Publ., 1961, 208 p.).

Fisher R.A. The wave of advance of advantageous genes. Annual Eugenics. 1937, vol. 7, pp. 355-369.

Gilmore R. Catastrophe Theory for Scientists and Engineers. New York, Wiley, 1981, 666 p. (Russian edition: Gilmor R. Prikladnaya teoriya katastrof [Applied Catastrophe Theory]. Moscow, Mir Publ., 1984, 350 p.)

Goldstein M.L. Major unsolved problems in space plasma physics. Astrophys. and Space Sci. 2001, vol. 277, no. $1 / 2$, pp. $349-369$.

Gradshteyn I.S., Ryzhik I.M. Tablitsy integralov, summ, ryadov i proizvedenii. [Tables of Integrals, Sums, Series, and Products]. Moscow, Fizmatgiz Publ., 1962, 1100 p. (In Russian). (English edition: Gradshteyn I.S., Ryzhik I.M. Tables of Integrals, Sums, Series, and Products. Academic Press, Elsevier Inc., 2007, 1171 p.).

Guglielmi A.V., Klain B.I., Rusakov N.N. Geoelectric field from observation at drift station North Pole - 22. Geo-fizicheskii sbornik AN UkSSR [Collected Papers on Geophysics of AS UkSSR].1979, iss. 88, pp. 60-64. (In Russian).

Guglielmi A.V. Problems in phenomenological modeling of $D_{\mathrm{st}}$ variation. Geomagnetizm $i$ aeronomiya [Geomagnetism and Aeronomy]. 1988, vol. 28, no. 2, pp. 272-276. (In Russian). 
Guglielmi A.V., Poyushkina T.N., Potapov A.S. Search for optimal source shape in RBM model of $D_{\text {st }}$ variation. Issledovaniya po geomagnetizmu, aeronomii i fizike Solntsa [Studies in Geomagnetism, Aeronomy and Solar Physics]. 1989, iss. 85, pp. 95-100. (In Russian).

Guglielmi A.V., Pokhotelov O.A. Geoelectromagnetic Waves. Bristol and Philadelphia, IOP Publ. Ltd., 1996, 402 p.

Guglielmi A.V. On threshold phenomena in geoelectrics. Fizika Zemli [Physics of the Earth]. 2002, no. 4, pp. 75-77. (in Russian).

Horsthemke W., Lefever R. Noise-Induced Transitions. Berlin, Springer, 1984, 299 p.

Kangas J., Guglielmi A., Pokhotelov O. Morphology and physics of short-period magnetic pulsations (A Review). Space Sci. Rev. 1998, vol. 83, pp. 435-512.

Kolmogorov A.N., Petrovsky N.G., Piskunov N.S. Studying the equation of diffusion combined with amount of a substance increase, and its application to one biological problem. Byulleten' MGU. Ser. A: Matematika $i$ mekhanika [Bull. MSU. Ser. A: Mathematics and Mechanics]. 1937, vol. 1, no. 6, pp. 1-16. (In Russian).

Landau L.D., Lifshits E.M. Statisticheskaya fizika [Statistical physics]. Part 1. Moscow, Fizmatlit Publ., 2005, 616 p. (In Russian). (English edition: Landau L.D., Lifshitz E.M. Statistical Physics. Part 1. Oxford, ButterworthHeinemann, 1980, 564 p.).

Migdal A. B. Kachestvennye metody v kvantovoi teorii polya [Qualitative Methods in Quantum Field Theory]. Moscow, Nauka Publ., 1975, 336 p. (In Russian). (English edition: Migdal A. B. Qualitative Methods in Quantum Theory. Addison-Wesley Publ., 1977, 437 p.)

Nishida A. Geomagnetic Diagnosis of the Magnetosphere. Springer-Verlag, 1978, 256 p. (Physics and Chemistry in Space, Book 9). (In English). (Russian edition: Nishida A. Geomagnitnyi diagnoz magnitosfery [Geomagnetic Diagnosis of the Magnetosphere]. Moscow, Nauka Publ., 1980, 299 p.).

Omori F. On after-shocks. Rep. Imp. Earthq. Inv. Corn. 1894, vol. 2, pp. 103-138.

Russell C.T., Hoppe M.M. Upstream waves and particles. Space Sci. Rev. 1983, vol. 34, no. 2, pp. 155-172. 